\title{
Programas de mentoria e promoção do desenvolvimento positivo de adolescentes
}

\author{
Eliana Silva ${ }^{\mathrm{i}}$ \& Teresa Freire ${ }^{\mathrm{ii}}$ \\ Universidade do Minho, Portugal
}

\begin{abstract}
Resumo
O conceito de mentoria tem sido aplicado de forma crescente na implementação de programas para o desenvolvimento de adolescentes em diversos contextos. De entre estes, destacam-se os contextos escolar e comunitário pela relevância na trajetória de vida. Da crescente popularidade destes programas tem surgido a necessidade de integração de teoria e investigação, para que cumpra os seus objetivos de cultivar e suportar relações de cuidado entre jovens e adultos não parentais. Neste artigo será apresentada uma nova abordagem do desenvolvimento de adolescentes, a perspetiva do desenvolvimento positivo, e a sua justificação como orientação teórica no desenvolvimento dos programas de mentoria. Esta perspetiva, por oposição ao tradicional foco na resolução de trajetórias não adaptativas, conceptualiza os jovens como dotados de potencialidades e recursos a desenvolver. Neste sentido, é apresentada evidência que fundamenta como a relação de adolescentes com adultos cuidadores e pares responsáveis, os mentores, pode contribuir para o desenvolvimento positivo.
\end{abstract}

Palavras-chave

Mentoria; Adolescentes; Desenvolvimento positivo

\section{Introdução}

O termo mentoria, conceptualizado como a partilha de conhecimento e aprendizagem intergeracional, apresenta uma longa história datando de 800 
a.c.. Foi primeiramente referenciado na mitologia grega da Odisseia, em que o mentor representava um amigo de confiança do rei de Ítaca. Esta imagem de um indivíduo mais velho que proporciona suporte e orientação, o mentor, permaneceu então até à atualidade (DuBois \& Karcher, 2005).

A aplicação do referido termo tem sido realizada segundo um caráter informal, através da relação estabelecida com adultos que naturalmente desempenham o papel de mentor em diversos contextos e atividades (e.g., nas escolas, no desporto) (DuBois \& Silverthorn, 2005a). Decorrente da verificação dos benefícios proporcionados por estas relações, o conceito tem vindo a ser aplicado na realização de programas formais. A literatura apresenta três tipos de programas - mentoria com adolescentes, académica e em contexto de trabalho -em resposta a diferentes necessidades desenvolvimentais dos indivíduos ao longo da vida (Eby, Allen, Evans, $\mathrm{Ng}$, \& DuBois, 2008). A mentoria com adolescentes apresenta como objetivo a promoção do seu desenvolvimento bem sucedido (Rhodes, 2002). A mentoria académica consiste na orientação proporcionada por adultos ou pares mais experientes a nível académico (e.g., melhoria das classificações) e não académico (e.g., suporte emocional) (Jacobi, 1991). Por sua vez, a mentoria profissional ocorre em contexto de trabalho com o propósito de crescimento pessoal e profissional (Kram, 1985).

Especificamente, a realização de programas com adolescentes tem verificado uma crescente aplicação nas últimas duas décadas, constituindo uma das intervenções sociais mais importantes (Herrera, Grossman, Kauh, \& McMaken, 2011). Por exemplo, nos Estados Unidos da América, mais de 5000 programas de mentoria são frequentados por cerca de três milhões de jovens, com 2.5 milhões de mentores (DuBois, Portillo, Rhodes, Silverthorn, \& Valentine, 2011). Programas e iniciativas semelhantes estão a surgir em todo o mundo (DuBois \& Karcher, 2005), conferindo uma dimensão global aos reconhecidos benefícios associados aos processos de mentoria.

$\mathrm{Na}$ base da crescente expansão desta intervenção com adolescentes está o reconhecimento de que relações positivas com adultos não parentais constituem um fator promotor de resiliência e de desenvolvimento normativo e saudável (Bowers et al., 2012; Rhodes, 2002). Em geral, os adolescentes são recetivos à presença de adultos não parentais dada a sua necessidade de independência e autonomização face à sua ligação aos pais, mas num 
momento em que ainda valorizam os conselhos dos indivíduos mais experientes (Grossman \& Bulle, 2006). Contudo, e no atual contexto sociohistórico, diversas mudanças nas sociedades e respetivas famílias têm reduzido significativamente o acesso dos jovens a estes adultos cuidadores (Simões \& Alarcão, 2011). Por estes motivos, tem-se verificado um interesse progressivo na implementação e procura dos programas de mentoria, acompanhado por um aumento da investigação científica dedicada aos processos e resultados que suportam a sua eficácia.

Neste trabalho pretende-se clarificar o conceito de mentoria, bem como o seu papel na promoção do desenvolvimento de adolescentes, de acordo com novas abordagens da literatura psicológica, nomeadamente a perspetiva do desenvolvimento positivo. Após a definição do conceito de mentoria e o enquadramento das suas principais características, pretende-se apresentar esta nova abordagem do desenvolvimento e a sua fundamentação como orientação para o desenho e implementação destes programas. Será ainda reunido um conjunto de evidência teórica e empírica acerca da eficácia da mentoria em dois dos principais contextos estruturantes do percurso de vida de adolescentes, como são a escola e a comunidade. Procura-se refletir sobre quais as condições de implementação que maximizam a sua eficácia ao nível do desenvolvimento de jovens saudáveis e felizes, quer no momento atual, quer no futuro, de adultos que possam contribuir ativamente para a sua sociedade.

\section{Conceito de mentoria}

Apesar da crescente investigação nesta área, várias definições de mentoria são ainda apresentadas na literatura científica. Por exemplo, Jacobi (1991), numa revisão da literatura, listou quinze diferentes definições. A principal razão apontada para a inexistência de uma definição universal tem sido associada ao facto de a mentoria constituir uma relação social, i.e., ocorre sempre num contexto e entre indivíduos específicos com diferentes características individuais, geralmente sobre a forma de programa (programas de mentoria). Assim, a imposição de definições específicas para todas as investigações não poderia reconhecer adequadamente as complexidades inerentes à sua aplicação real (Allen \& Eby, 2007). 
O debate relativo à definição destes programas de mentoria cria um desafio à tentativa de clarificar o seu impacto e as diferentes práticas que contribuem para atingir os objetivos estabelecidos e subjacentes ao conceito. Contudo, ao longo das últimas três décadas, o conceito de mentoria tem sido mais desenvolvido, permitindo a emergência de uma maior clarificação ao nível da sua definição e, igualmente, a aceitação da sua diversidade dependendo dos objetivos e aplicações da intervenção (Passmore, Peterson, \& Freire, 2013). Concretamente, em relação a programas com adolescentes, existe consenso de que se trata de um tipo de intervenção cujo objetivo é promover resultados desenvolvimentais positivos em diferentes áreas do funcionamento (e.g., emocional, comportamental, académico) através de uma relação duradoira de suporte e cuidado entre indivíduos jovens (mentorandos) e adultos não parentais (mentores) (DuBois et al., 2011; DuBois, Holloway, Valentine, \& Cooper, 2002).

De acordo com a estrutura tradicional dos programas com adolescentes, o mentor é um voluntário adulto da comunidade em interação regular apenas com um jovem durante um período mínimo de um ano. Nos últimos anos, tem-se verificado uma maior complexidade, nomeadamente a sua realização com grupos específicos (e.g., estudantes em risco de baixo desempenho académico/abandono escolar), com alvo em determinados resultados (e.g., realização académica, prevenção de comportamentos de risco), em diferentes contextos (e.g., escolas, hospitais), e/ou utilizando formatos e modelos alternativos (e.g., programas em grupo, por pares, online e mentoria intergeracional) (DuBois et al., 2011).

Dada a diversidade e complexidade crescentes na aplicação do conceito de mentoria com adolescentes em crescimento, torna-se fundamental que a implementação dos programas seja orientada pela teoria e investigação acerca do desenvolvimento na adolescência de forma a melhorar os processos e resultados desta intervenção.

\section{Mentoria e promoção do desenvolvimento de adolescentes}

\subsection{O desenvolvimento positivo de adolescentes}

Até ao início da década de 80/90, a conceptualização predominante na literatura acerca do estudo científico da adolescência fundamentava-se em 
perspetivas teóricas caracterizadas por uma ideia de "défice". Este período do ciclo de vida era então descrito como um período inevitável de "tumulto e stress" (Hall, 1904), com a consequente emergência de perturbações do desenvolvimento (Freud, 1969). Esta perspetiva conceptualizava os adolescentes como "problemas a serem resolvidos" (Roth \& Brooks-Gunn, 2003), focalizando-se em trajetórias de desenvolvimento não adaptativas. As estratégias para melhorar as suas vidas centravam-se no tratamento e prevenção de comportamentos de risco (Mueller et al., 2011).

Nas últimas décadas, uma nova área da Psicologia - a Psicologia Positiva, ou "psicologia do funcionamento ótimo" (Csikszentmihalyi, 2011; Seligman \& Csikszentmihalyi, 2000) - constituiu-se como uma abordagem de grande relevância na melhoria da qualidade de vida das sociedades modernas (Donaldson, 2011). A psicologia positiva apresenta como foco de estudo os aspetos positivos do funcionamento humano, considerando que a ausência de doença, embora necessária e desejável, não é em si suficiente para a concretização de uma vida plena e saudável (Freire, 2009; Seligman \& Csikszentmihalyi, 2000).

$\mathrm{Na}$ linha desta nova perspetiva na conceptualização do funcionamento humano, surgiu, no início do século XXI, uma nova visão e mesmo vocabulário na discussão do desenvolvimento da adolescência, como sejam: bem-estar, envolvimento cívico, recursos desenvolvimentais, potencial futuro e desenvolvimento positivo (Benson, 2003; Freire, 2013; Lerner, Alberts, Jelicic, \& Smith, 2006). Esta perspetiva considera os jovens como agentes do seu próprio crescimento, possuindo um potencial natural e normativo para um desenvolvimento saudável e bem sucedido. Assim, e em termos gerais, o desenvolvimento positivo dos jovens refere-se ao envolvimento em comportamentos pró-sociais e evitamento de comportamentos que comprometem e prejudicam a saúde e o futuro (Freire \& Soares, 2000). Este desenvolvimento ocorre na dinâmica relacional de adolescentes com os contextos, relações e interações que constituem a vida diária, incluindo a comunidade, a escola, a família e os pares (Larson, 2006).

$O$ foco no estudo das características positivas e adaptativas da adolescência tem sido integrado em vários modelos teóricos designados por modelos do desenvolvimento positivo de adolescentes (Larson, 2006; Lerner, Brentano, Dowling, \& Anderson, 2002). Uma das abordagens mais 
amplamente referenciadas é o modelo dos recursos desenvolvimentais, considerados bases universais do desenvolvimento positivo (Benson, 2003). Foi identificado um conjunto de recursos individuais referentes a qualidades pessoais dos jovens (e.g., valores positivos, competências sociais) e recursos contextuais relativos à necessidade de acesso a locais seguros, atividades desafiadoras e cuidado dos outros (e.g., casa, escola, comunidade). Estes recursos devem constituir-se como alvo de intervenção para o desenvolvimento de trajetórias de vida positivas, o que se pode traduzir na alteração dos contextos diários de forma a reduzir fatores de risco ou aumentar a experiência de suporte externo, em associação com a promoção dos recursos individuais dos jovens (Larson, 2006).

Destaca-se ainda o modelo de Lerner e colaboradores (2002), que atualmente constitui a abordagem teórica com maior evidência empírica (Bowers et al., 2010). O modelo apresenta os cinco resultados desejáveis do desenvolvimento positivo, ou "Cinco C's": competência - visão positiva das suas ações em diversos domínios (e.g., social, académico, cognitivo e vocacional); confiança - perceção de valor próprio e autoeficácia; conexão relações sociais positivas; caráter - respeito pelas normas sociais e culturais, moralidade e integridade; e cuidado/compaixão - sentimento de empatia e simpatia pelos outros. Quando todos estes elementos estão presentes na vida de um adolescente, emerge um sexto " $C$ " do desenvolvimento positivo: a contribuição para o self, família e comunidade.

Os modelos apresentados têm sido aplicados como orientação teórica no desenho e implementação de programas de intervenção para o desenvolvimento positivo.

\subsection{Programas de intervenção}

A investigação tem verificado a importância da intervenção para a promoção do desenvolvimento não porque existem problemas a resolver, segundo a conceptualização tradicional de adolescência, mas apenas porque existem adolescentes em crescimento e desenvolvimento (Freire \& Silva, in press; Freire \& Soares, 2000). Apesar de os jovens apresentarem potencialidades para se constituírem agentes do seu próprio crescimento, este percurso deve ser apoiado e suportado por adultos que orientem as suas 
trajetórias de vida, desenvolvendo recursos e competências promotoras do bem-estar na vida diária (Larson, 2006).

Como orientação teórica para a implementação destas intervenções, foram identificadas três características, designadamente os objetivos, o ambiente do programa e as atividades realizadas. Ao nível dos objetivos destaca-se a promoção do desenvolvimento positivo e a preparação para o futuro, mesmo quando há o objetivo de prevenção de comportamentos de risco. Os programas devem proporcionar um ambiente positivo, física e psicologicamente seguro, com expectativas de sucesso, responsabilidade e compromisso. Finalmente, ao nível das atividades realizadas é importante criar oportunidades para o desenvolvimento de interesses, talentos, com a possibilidade de praticar novas competências (Roth \& Brooks-Gun, 2003).

A literatura tem identificado um conjunto de programas que integram estes pressupostos na sua implementação, destacando-se no presente artigo os programas de mentoria que, pelas suas características, se constituem como uma intervenção válida e eficaz na promoção do desenvolvimento positivo de adolescentes.

\subsubsection{Programas de mentoria com adolescentes}

Os programas de mentoria com adolescentes consistem numa intervenção preventiva e promotora do desenvolvimento, apresentando como principal componente a relação estabelecida entre o jovem e o mentor (Rhodes, 2002). Ao longo do tempo, a investigação tem-se dedicado à explicação dos processos através dos quais relações positivas mentormentorando contribuem para melhorias em determinadas dimensões psicológicas e comportamentais. Neste sentido, Rhodes $(2002,2005)$ propôs um modelo conceptual segundo o qual os mentores influenciam os resultados desenvolvimentais em três domínios inter-relacionados: competências sociais e bem-estar emocional; competências cognitivas; e desenvolvimento positivo da identidade. Através do suporte consistente ao longo do tempo, os mentores podem desafiar visões negativas que os jovens possam ter de si próprios e dos outros e demonstrar que o estabelecimento de relações positivas com adultos é possível. Neste sentido, a relação com o mentor pode constituir uma experiência emocional adaptativa e saudável que se pode generalizar a 
outras relações sociais, melhorando as competências interpessoais no dia-adia (Chan et al., 2013; Rhodes, 2002, 2005). A investigação tem fornecido evidência em suporte destes processos, verificando-se melhorias nas perceções dos adolescentes acerca das relações com os seus pares e adultos significativos (e.g., com os pais) ao nível da intimidade, comunicação e confiança experienciadas (Chan et al., 2013; DuBois et al., 2002).

A relação com o mentor pode contribuir para o desenvolvimento cognitivo dos jovens através da exposição a novas oportunidades de aprendizagem, desafio intelectual e orientação, bem como promoção de sucesso académico (Rhodes, 2002, 2005). O estabelecimento de uma relação de qualidade pode favorecer a participação em atividades, criando oportunidades educacionais e ocupacionais que podem informar as decisões e comportamentos atuais e futuros dos jovens e contribuir para o desenvolvimento de um sentido de identidade individual e social (DuBois et al., 2011).

Estes processos têm sido identificados como os principais resultados promovidos pelos programas de mentoria. Contudo, a implementação em contextos específicos surge associada ao desenvolvimento de diferentes competências.

\subsubsection{Contextos de implementação: a comunidade e a escola}

Têm sido identificados como principais contextos de implementação dos programas de mentoria a escola e a comunidade envolvente, pela relevância que assumem na vida diária de adolescentes (Benson, 2003; Lima \& Freire, 2009). Em ambos os contextos, verifica-se um aumento da prevalência dos programas; contudo, a aplicação nas escolas tem conhecido um crescimento mais significativo do que nas comunidades, constituindo cerca de $70 \%$ dos programas de mentoria nos Estados Unidos da América (Chan et al., 2013; Herrera et al., 2011).

Tradicionalmente, os programas de mentoria têm sido implementados em contexto comunitário, a partir do reconhecimento teórico e empírico de que a participação em programas neste contexto constitui o recurso desenvolvimental que parece ter uma maior influência para o desenvolvimento positivo de adolescentes (Scales, Benson, Leffert, \& Blyth, 
2000). A investigação tem fornecido suporte para a sua aplicação, verificando efeitos positivos ao nível da prevenção de comportamentos de risco e promoção de competências sociais e resultados académicos (DuBois et al., 2002).

A aplicação da mentoria em contexto escolar surgiu do aumento da preocupação e dos esforços destas instituições para implementar intervenções que possam lidar com os desafios desenvolvimentais e académicos dos estudantes, apresentando como objetivo último a promoção do sucesso académico (Herrera et al., 2011; Simões \& Alarcão, 2011). Evidência empírica tem reforçado a sua importância, demonstrando que a criação de ambientes de aprendizagem apoiantes, com ambientes, pessoas e oportunidades positivas contribui para a saúde mental e o desenvolvimento de competências (Portwood \& Ayers, 2005).

A investigação tem comparado a aplicação tradicional dos programas de mentoria em contexto comunitário com a sua aplicação nas escolas de forma a verificar se os programas nestes diferentes contextos se revelam igualmente capazes de contribuir para o desenvolvimento positivo de adolescentes. Por um lado, os programas nas escolas apresentam limitações inerentes ao contexto, como a realização de menos encontros entre o mentor e os jovens e uma menor duração global do programa devido aos constrangimentos do calendário letivo (King, Vidourek, Davis, \& McClellan, 2002; Portwood \& Ayers, 2005). Estes fatores, possivelmente, apresentam efeitos ao nível das relações desenvolvidas com os mentores, caracterizadas pelos participantes como de menor proximidade comparativamente com as relações referidas em contexto comunitário (Grossman \& Bulle, 2006). Por outro lado, têm sido destacadas vantagens do contexto escolar, o que inclui baixos custos, maior facilidade de acesso aos jovens e maior estrutura e supervisão no decurso do programa (Portwood \& Ayers, 2005). A especificidade das interações presentes em contexto escolar permite ainda que a relação entre o jovem e o seu mentor contribua para melhorias em diversas áreas de funcionamento (e.g., académico, social e comportamental), particularmente relevantes em contexto escolar.

A investigação, de facto, tem sugerido que os programas de mentoria implementados em escolas apresentam benefícios para os jovens num período desenvolvimental em que se cruza a necessidade de suporte 
académico e relacional (Herrera et al., 2011). Têm sido observadas melhorias ao nível do desempenho académico (Herrera et al., 2011; King et al., 2002), autoeficácia percebida (Herrera et al., 2011), atitudes em relação à escola (King et al., 2002), melhorias na autoestima e nas relações com os pares e adultos significativos, como pais e professores (Chan et al., 2013; King et al., 2002). Tendo em conta a evidência crescente da eficácia da aplicação dos programas em contexto escolar ao nível da promoção de competências académicas e pessoais, observa-se nos últimos anos a integração de formatos alternativos, com destaque do conceito de mentoria por pares.

\section{Programas de mentoria por pares: promoção do sucesso académico ao longo do desenvolvimento}

Para além dos benefícios apresentados, decorrentes dos programas de mentoria com adultos, a investigação tem demonstrado que os programas com base nas relações entre pares também se revelam eficazes. De facto, há estudos empíricos significativos que demonstram os benefícios da aprendizagem colaborativa e cooperativa entre pares (Ladyshewsky, 2006). Estes programas de intervenção, designados de mentoria por pares, ajuda de pares ou tutoria por pares, apresentam em comum a colaboração e cooperação entre pares e têm constituído uma prática integrante de diferentes instituições e níveis de ensino.

Ao nível do ensino básico e secundário, os pressupostos desta intervenção são aplicados essencialmente através de um acompanhamento académico de alunos que apresentam dificuldades específicas, sendo o acompanhamento realizado por alunos com melhor aproveitamento nessas áreas ou de outro nível de escolaridade (MENTOR, 2005). Ambos os intervenientes parecem beneficiar desta relação, nomeadamente no que se refere à autoestima e maturação psicossocial (Pyatt, 2002).

Também no contexto universitário o conceito de mentoria por pares tem sido aplicado de forma crescente, não só com propósitos estritamente académicos como também ao nível da integração bem sucedida dos estudantes (Heirdsfield, Walker, Walsh, \& Wilss, 2008; Jacobi, 1991). Segundo Beasley (1997), "alguns estudantes fazem uma transição suave e rápida dos papéis e padrões do ensino secundário, tornando-se aprendizes 
independentes e pensadores críticos, enquanto outros batalham ao longo das suas carreiras universitárias". De facto, na transição para o ensino superior, os estudantes enfrentam determinados desafios desenvolvimentais (e.g., aumento da independência) e, por vezes, sentimentos de isolamento e incerteza com implicações na integração/adaptação ao novo contexto de ensino e percurso académico (Heirdsfield et al., 2008). Neste sentido, a implementação de programas de mentoria por pares consiste na orientação e suporte proporcionados por estudantes mais experientes aos novos colegas, no que toca ao seu desenvolvimento profissional (e.g., desenvolvimento de competências académicas) e psicossocial (e.g., suporte emocional e psicológico). A investigação tem fornecido evidência em favor da sua implementação, através da identificação de resultados positivos para os mentorandos (e.g., melhorias no desempenho académico e integração social), mentores (e.g., desenvolvimento de relações interpessoais, satisfação com o papel desempenhado) e universidades (e.g., redução das taxas de abandono) (Eby et al., 2008; Heirdsfield et al., 2008).

Dado o reconhecido potencial destes programas, têm sido aplicados em diferentes instituições de ensino superior, sobretudo a nível internacional. Em Portugal verifica-se ainda uma escassez, quer ao nível da sua aplicação quer ao nível da investigação dos seus processos. Destaca-se um projeto pioneiro na Universidade do Minho, designado de "Tutorias por Pares", cuja implementação foi realizada pela primeira vez no ano letivo de 2006-2007. O programa pretende contribuir para o sucesso académico dos novos estudantes através da promoção da sua integração global no contexto universitário. Assim, através da colaboração entre pares, é disponibilizado apoio aos colegas que chegam pela primeira vez ao ensino superior, proporcionando conhecimentos importantes para a sua integração (e.g., funcionamento da instituição, organização espacial do campus, serviços disponíveis).

Entre os principais procedimentos do programa, destaca-se a participação voluntária dos estudantes. Os tutores atendem os tutorandos em pequenos grupos de dois a cinco elementos, em tempo e espaços próprios, no campus da Universidade, semanalmente ou quinzenalmente, durante aproximadamente 60 minutos. Vários tópicos são abordados, de forma a responder às necessidades e dúvidas relativas à sua integração. Toda a 
implementação do programa tem a supervisão da equipa coordenadora (Freire, 2012).

Uma investigação realizada de forma a conhecer e compreender as perceções dos estudantes em relação aos papéis desempenhados (Freire, 2012) revelou que os participantes identificaram ganhos significativos decorrentes da participação, nomeadamente ao nível do desenvolvimento de competências pessoais e académicas. Os estudantes consideraram ainda que se sentem mais integrados no contexto universitário, melhoraram o seu desempenho e o envolvimento social.

Os resultados demonstram a importância do investimento neste tipo de apoio aos estudantes no atual contexto universitário, em que os objetivos de ensino e aprendizagem estão relacionados com a aquisição de competências que vão para além das questões curriculares, o que significa que o sucesso académico também está associado à integração social. Da mesma forma, conceptualizando a aprendizagem como um processo social partilhado, a experiência de integrar um grupo de pares torna-se uma oportunidade de melhorar comportamentos e práticas pró-sociais. Assim, considerando o contexto universitário como um processo inacabado em termos do desenvolvimento de competências dos estudantes, justifica-se a integração destes programas na trajetória académica (Freire, 2012).

\section{Componentes para a eficácia dos programas de mentoria}

A par da crescente aplicação do conceito de mentoria em diferentes formatos e contextos, torna-se relevante e necessário conhecer as condições sob as quais os programas podem cumprir os seus objetivos e ter um impacto positivo e transformador na vida dos jovens. Neste sentido, foram estabelecidos critérios com base na evidência (MENTOR, 2009), com integração da pespetiva do desenvolvimento positivo, a considerar na implementação dos programas, que constituem "um fenómeno complexo e multifacetado" (DuBois \& Silverthorn, 2005b, p. 44) que requer uma cuidadosa planificação, implementação, monitorização e avaliação. Destacam-se, assim, seis dimensões fundamentais: recrutamento, seleção, orientação/formação inicial, compatibilização, acompanhamento/monitorização e finalização. 
O primeiro critério relaciona-se com o processo de recrutamento dos participantes (mentores e mentorandos). Em relação aos mentores, é fundamental realizar uma descrição realista acerca do programa no que concerne aos seus benefícios, objetivos, resultados esperados e possíveis desafios. No processo de recrutamento dos jovens é necessário verificar se os serviços oferecidos correspondem às suas necessidades e apresentar os procedimentos desta intervenção. A ausência destas componentes no início do programa tem sido associada ao abandono ou a uma finalização precoce e, consequentemente, ao surgimento de efeitos indesejáveis para os jovens participantes (e.g., diminuição da autoestima e da confiança nos adultos) (MENTOR, 2009).

O segundo critério consiste no processo de seleção dos participantes. Para o desempenho do papel de mentor é necessário verificar a presença de uma série de requisitos considerados indispensáveis para a realização das suas funções com responsabilidade e qualidade (e.g., disponibilidade temporal de acordo com o tempo atribuído ao exercício da mentoria). Este processo permite determinar a sua aptidão e assegurar a segurança física e psicológica dos jovens, aspetos que justificam a sua inclusão em qualquer programa. Para a participação dos jovens é necessária a autorização dos seus pais (MENTOR, 2009).

Após estes procedimentos ao nível da seleção, é necessário fornecer orientação e formação inicial, sobretudo aos mentores, o que constitui o terceiro critério. $O$ objetivo é promover conhecimentos e competências para $o$ desenvolvimento de uma relação adequada com os mentorandos. Alguns dos conteúdos a abordar incluem os procedimentos e objetivos do programa, perspetivas do desenvolvimento de adolescentes e questões éticas. Se apropriado, de acordo com os propósitos do programa, é fornecida alguma formação aos jovens e seus pais acerca das regras de funcionamento, deveres e papéis dos mentores e mentorandos. A presença desta componente está associada a uma maior satisfação dos participantes e ao estabelecimento de relações mais positivas mentor-mentorando, contribuindo para a eficácia global do programa (MENTOR, 2009).

O quarto critério refere-se à utilização de mecanismos de compatibilização (matching) entre mentores e mentorandos, dado que a investigação tem demonstrado que as relações estabelecidas apresentam 
maior probabilidade de serem eficazes e de maior duração quando os elementos são associados de acordo com determinadas dimensões (e.g., interesses mútuos). $O$ quinto critério relaciona-se com 0 acompanhamento/monitorização e suporte providenciados ao longo do programa que contribuem para uma participação mais satisfatória e uma maior permanência dos participantes. Contudo, a finalização do programa, o sexto e último critério, constitui uma parte inevitável que é necessário ter em consideração. Destaca-se a importância de estabelecer políticas e procedimentos para lidar com as finalizações de uma forma consistente e cuidada. Neste sentido, a literatura recomenda a realização de uma atividade ou evento em que mentor e mentorando possam participar e que represente o final do programa e da relação estabelecida, para além da avaliação das suas experiências, por exemplo através de entrevistas (e.g., refletir acerca das experiências partilhadas e do seu impacto) (MENTOR, 2009).

Para além destes critérios, têm sido identificados elementos adicionais a ter em consideração nos programas de mentoria, com destaque para a participação da família (King et al., 2002). Verifica-se uma variedade significativa, entre os procedimentos de vários programas, em relação ao tipo e quantidade de envolvimento parental. Embora a participação da família não constitua uma prática recorrente, o que está em causa é perceber de que forma as relações positivas entre os mentores e mentorandos podem moldar melhorias nas relações que compõem a dinâmica familiar. Da mesma forma, se os pais se sentirem envolvidos nos programas em que outros adultos proporcionam suporte aos seus filhos, a probabilidade de reforçarem as suas influências positivas é maior (DuBois \& Karcher, 2005). Neste sentido, é recomendado o estabelecimento de relações colaborativas entre os mentores e os pais, fornecendo informação acerca do programa e do progresso dos jovens (Chan et al., 2013).

\section{Conclusão}

A adolescência constitui um período do ciclo de vida em que se vão definindo e moldando diferentes trajetórias. Neste artigo, através de uma abordagem centrada nas potencialidades, pretendeu-se destacar a importância das relações de suporte estabelecidas com adultos não parentais e pares responsáveis para a definição positiva destas trajetórias 
desenvolvimentais. Apesar da sua presença, mais ou menos constante na vida diária de adolescentes, a intencionalização destas relações através da criação de programas de mentoria tem-se revelado eficaz ao nível da prevenção do envolvimento em comportamentos de risco e da promoção de um desenvolvimento bem sucedido na escola, na comunidade e na vida em geral (DuBois et al., 2011).

Estes resultados sugerem a eficácia da mentoria; contudo, é necessário ter em atenção que uma intervenção isolada não se revela capaz de transformar a vida de adolescentes. A maioria dos jovens necessita de uma sucessão de experiências e intervenções positivas, formais e informais. De facto, há evidência de que estes programas conduzem a maiores benefícios quando acompanhados de outros serviços de suporte (Eby et al., 2008). Assim, para que esta intervenção continue a promover resultados positivos e cada vez mais adolescentes possam beneficiar da sua participação, teoria e investigação necessitam de assumir um papel mais central, de forma a esclarecer as condições que maximizam a sua eficácia e o seu potencial efetivo na promoção de adolescentes bem sucedidos no presente e adultos responsáveis no futuro.

\section{Referências}

Allen, T., \& Eby, L. (Eds.). (2007). Blackwell handbook of mentoring: A multiple perspectives approach. London, England: Wiley-Blackwell.

Beasley, C. (1997). Students as teachers: The benefits of peer tutoring. In R. Pospisil \& L. Willcoxson (Eds.), Learning through teaching. Proceedings of the 6th Annual Teaching Learning Forum (pp. 21-30). Perth: Murdoch University. Disponivel em: http://lsn.curtin.edu.au/tlf/tlf1997/beasley.html

Benson, P. L. (2003). Developmental assets and asset-building community: Conceptual and empirical foundations. In R. M. Lerner \& P. L. Benson (Eds.), Developmental assets and asset-building communities: Implications for research, policy, and practice (pp. 19-43). New York: Kluwer Academic/Plenum.

Bowers, E. P., Geldhof, G. J., Schmid, K. L., Napolitano, C. M., Minor, K., \& Lerner, J. V. (2012). Relationships with important nonparental adults and positive youth development: An examination of youth self-regulatory strengths as mediators. Research in Human Development, 9(4), 298-316.

Bowers, E. P., Li, Y., Kiely, M. K., Brittian, A., Lerner, J. V., \& Lerner, R. M. (2010). The five Cs model of positive youth development: A longitudinal analysis of confirmatory factor structure and measurement invariance. Journal of Youth and Adolescence, 39, 720-735. 
Chan, C. S., Rhodes, J. E., Howard, W. J., Lowe, S. R., Schwartz, S. E., \& Herrera, C. (2013). Pathways of influence in school-based mentoring: The mediating role of parent and teacher relationships. Journal of School Psychology, 51, 129-142.

Csikszentmihalyi, M. (2011). Positive psychology and positive world-view: New hope for the future of humankind. In S. I. Donaldson, M. Csikszentmihalyi \& J. Nakamura (Eds.), Applied positive psychology. Improving everyday life, health, schools, work and society (pp. 205-211). New York: Psychology Press.

Donaldson, S. I. (2011). Determining what works, if anything, in positive psychology. In S. I. Donaldson, M. Csikszentmihalyi \& J. Nakamura (Eds.), Applied positive psychology improving everyday life, health, schools, work, and society (pp. 311). New York: Routledge.

DuBois, D. L., \& Karcher, M. J. (2005). Youth mentoring: Theory, research, and practice. In D. L. DuBois \& M. J. Karcher (Eds.), Handbook of youth mentoring (pp. 2-11). Thousand Oaks, CA: Sage.

DuBois, D. L., \& Silverthorn, N. (2005a). Natural mentoring relationships and adolescent health: Evidence from a national study. American Journal of Public Health, 95, 518-524.

DuBois, D. L., \& Silverthorn, N. (2005b). Research methodology. In D. L. DuBois \& M. J. Karcher (Eds.), Handbook of youth mentoring (pp. 44-64). Thousand Oaks, CA: Sage.

DuBois, D., Holloway, B., Valentine, J., \& Cooper, H. (2002). Effectiveness of mentoring programs for youth: A meta-analytic review. American Journal of Community Psychology, 2(30), 157-197.

DuBois, D., Portillo, N., Rhodes, J., Silverthorn, N., \& Valentine, J. (2011). How effective are mentoring programs for youth? A systematic assessment of the evidence. Psychological Science in the Public Interest, 12(2), 57-91.

Eby, L., Allen, T., Evans, S., Ng, T., \& DuBois, D. (2008). Does mentoring matter? A multidisciplinary meta-analysis comparing mentored and non-mentored individuals. Journal of Vocational Behavior, 72, 254-267.

Freire, T. (2009). Understanding positive life. Research and practice on positive psychology. Lisboa: Climepsi.

Freire, T. (2013). Leisure experience and positive identity development in adolescents. In T. Freire (Ed.), Positive leisure science: From subjective experience to social contexts. Dordrecht, NL: Springer.

Freire, T. (Org.). (2012). Tutoria por pares na Universidade do Minho: Manual para tutores. Braga: Universidade do Minho.

Freire, T., \& Silva, E. (in press). Adolescentes e desenvolvimento positivo: Para uma abordagem inovadora da adolescência. In T. Medeiros (Org.), Adolescência. Açores: Universidade dos Açores.

Freire, T., \& Soares, I. (2000). A intervenção na adolescência através do lazer e a promoção do desenvolvimento positivo. Psicologia, Educação e Cultura, IV(2), 329-343.

Freud, A. (1969). Adolescence as a developmental disturbance. In G. Caplan \& S. Lebovici (Eds.), Adolescence (pp. 5-10). New York: Basic Books. 
Grossman, J. B., \& Bulle, M. J. (2006). Review of what youth programs do to increase the connectedness of youth with adults. Journal of Adolescence Health, 39, 788799.

Hall, G. S. (1904). Adolescence: Its psychology and its relations to physiology, anthropology, sociology, sex, crime, religion, and education. New York: Appleton.

Heirdsfield, A. M., Walker, S., Walsh, K., \& Wilss, L. (2008). Peer mentoring for first year teacher education students: The mentors' experience. Mentoring \& Tutoring: Partnership in Learning, 16(2), 109-124.

Herrera, C., Grossman, J. B., Kauh, T. J., \& McMaken, J. (2011). Mentoring in schools: An impact study of Big Brothers Big Sisters school-based mentoring. Child Development, 82(1), 346-361.

Jacobi, M. (1991). Mentoring and undergraduate academic success: A literature review. Review of Educational Research, 4(61), 505-532.

King, K. A., Vidourek, R. A., Davis, B., \& McClellan, W. (2002). Increasing self-esteem and school connectedness through a multidimensional mentoring program. Journal of School Health, 72, 294-299.

Kram, K. E. (1985). Mentoring at work. Glenview, IL: Scott Foresman.

Ladyshewsky, R. (2006). Building cooperation in peer coaching relationships: Understanding the relationships between reward, structure, learner preparedness, coaching skill and learner engagement. Psychotherapy, 92, 4-10.

Larson, R. (2006). Positive youth development, willful adolescents, and mentoring. Journal of Community Psychology, 34, 677-689.

Lerner, R. M., Alberts, A. E., Jelicic, H., \& Smith, L. M. (2006). Young people as resources to be developed: Promoting positive youth development through adult-youth relations and community assets. In E. G. Clary \& J. E. Rhodes (Eds.), Mobilizing adults for positive youth development: Strategies for closing the gap between beliefs and behaviors (pp. 19-39). Minneapolis, MN: Search Institute.

Lerner, R., Brentano, C., Dowling, E., \& Anderson, P. (2002). Positive youth development: Thriving as the basis of personhood and civil society. New directions for youth development, 95, 11-33.

Lima, I., \& Freire, T. (2009). Qualidade da experiência subjetiva no quotidiano escolar de adolescentes: Implicações desenvolvimentais e educacionais. Análise Psicológica, 4, 523-534.

MENTOR/National Mentoring Partnership (2005). How to build a successful mentoring program using the elements of effective practice: A step-by-step toolkit for program managers. Alexandria, VA: MENTOR.

MENTOR/National Mentoring Partnership (2009). Elements of effective practice for mentoring (3rd ed.). Alexandria, VA: MENTOR.

Mueller, M. K., Phelps, E., Bowers, E. P., Agans, J. P., Urban, J. B., \& Lerner, R. M. (2011). Youth development program participation and intentional self-regulation skills: Contextual and individual bases of pathways to positive youth development. Journal of Adolescence, 34, 1115-1125. 
Passmore, J., Peterson, D., \& Freire, T. (2013). The psychology of coaching and mentoring. In J. Passmore, D. Peterson \& T. Freire (Eds.), The Wiley-Blackwell Handbook of the Psychology of Coaching \& Mentoring (pp. 3-11). Chichester: Wiley-Blackwell.

Portwood, S. G., \& Ayers, P. M. (2005). Schools. In D. L. DuBois \& M. J. Karcher (Eds.), Handbook of youth mentoring (pp. 336-347). Thousand Oaks, CA: Sage.

Pyatt, G. (2002). Cross-school mentoring: Training and implementing a peer mentoring strategy. Mentoring \& Tutoring, 10(2), 171-177.

Rhodes, J. E. (2002). Stand by me: The risks and rewards of mentoring today's youth. Cambridge, MA: Harvard University Press.

Rhodes, J. E. (2005). A model of youth mentoring. In D. DuBois \& M. Karcher (Eds.), Handbook of youth mentoring (pp. 30-43). Thousand Oaks, CA: Sage.

Roth, J., \& Brooks-Gunn, J. (2003). What exactly is a youth development program? Answers from research and practice. Applied Developmental Science, 7(2), 94111.

Scales, P. C., Benson, P. L., Leffert, N., \& Blyth, D. A. (2000).The contribution of developmental assets to the prediction of thriving among adolescents. Applied Developmental Science, 4, 27-46.

Seligman, M., \& Csikszentmihalyi, M. (2000). Positive psychology: An introduction. American Psychologist, 55(1), 5-14.

Simões, F., \& Alarcão, M. (2011). A eficácia da mentoria escolar na promoção do desenvolvimento sócioemocional e instrumental de jovens. Educação e Pesquisa, 2(37), 339-354. 


\title{
MENTORING PROGRAMS AND PROMOTION OF POSITIVE YOUTH DEVELOPMENT
}

\begin{abstract}
The concept of mentoring has been increasingly applied in programs aimed at adolescent development in different contexts. Among these, we highlight the school and community contexts because of its relevance in adolescents' life trajectory. Given the growing popularity of these programs, integration of theory and research is necessary to fulfill its goals of cultivating and supporting caring relationships between youth and nonparental adults. In this article we present a new approach to adolescents' development, the perspective of positive development, and its justification as theoretical foundation in the development of mentoring programs. This perspective, as opposed to the traditional focus on solving maladaptive trajectories, conceptualizes youth as having potentials and resources to develop. In this sense, we present evidence to support how the relationship of adolescents with caring adults and responsible peers, mentors, can contribute to the positive development.
\end{abstract}

Keywords

Mentoring; Adolescents; Positive development

PROGRAMMES DE MENTORAT ET LA PROMOTION DU DÉVELOPPEMENT POSITIF DES JEUNES

\section{Résumé}

Le concept de mentorat est de plus en plus appliqué dans les programmes visant le développement des adolescents dans des contextes différents. Nous mettons en évidence les contextes scolaires et communautaires en raison de sa pertinence dans la trajectoire de vie des adolescents. En ce qui concerne ces programmes, l'intégration de la théorie et de la recherche est nécessaire pour atteindre leurs objectifs de cultiver et soutenir les relations bienveillantes 
entre les jeunes et les adultes non parentaux. Nous présentons une nouvelle approche pour le développement des adolescents, la perspective d'un développement positif, et sa justification en tant que fondement théorique dans le développement de programmes de mentorat. On conceptualise les jeunes comme ayant des potentiels et des ressources pour se développer et nous présentons des preuves à l'appui comment la relation des adolescents avec des adultes bienveillants et des pairs responsables, les mentors, peuvent contribuer au développement positif.

Mots-clé

Mentorat; Adolescents; Développement positif 